Supporting information for:

\title{
Immunodetection of Lung IgG and IgM Antibodies Against SARS-CoV-2 via Enzymatic Liquefaction of Respiratory Samples from COVID-19 Patients.
}

Antonio Clemente, ${ }^{1 *}$ Alejandra Alba-Patiño, ${ }^{1,2, \$}$ Giulia Santopolo, $, 2, \$$ Estrella Rojo-Molinero, ${ }^{3}$ Antonio Oliver, ${ }^{3}$ Marcio Borges, ${ }^{1,4}$ María Aranda, ${ }^{1,4}$ Alberto del Castillo,,${ }^{1,4}$ and Roberto de la Rica. ${ }^{1,2 *}$

${ }^{1}$ Multidisciplinary Sepsis Group, Health Research Institute of the Balearic Islands (IdISBa), Palma de Mallorca, Spain.

2University of the Balearic Islands, Chemistry Department, Palma de Mallorca, Spain.

${ }^{3}$ Microbiology Department, Son Espases University Hospital, Health Research Institute of the Balearic Islands (IdISBa), Palma de Mallorca, Spain.

${ }^{4}$ Multidisciplinary Sepsis Unit, ICU, Son Llàtzer University Hospital, Palma de Mallorca, Spain.

\section{Contents:}

Video S1. Enzymatic liquefaction of BAS sample from a COVID-19 patient.

Figure S1. Optimization of secondary biotinylated antibodies to detect human IgM and IgG.

Figure S2. Impact of sample pretreatment by enzymatic method on immunodetection. 


\section{S1. ELISA for human IgM and IgG.}

ELISA protocol: the microtiter plate was coated with human IgG or IgM (both from Abcam) at different concentrations $\left(0,0.1,1,10,100,1000,10000\right.$ and $\left.100000 \mathrm{pg} \cdot \mathrm{mL}^{-1}\right)$ in bicarbonate buffer (0.1 M, pH 9.6). After overnight incubation at $4{ }^{\circ} \mathrm{C}$ the plate was washed 3 times with PBS containing $0.1 \%$ Tween 20 (PBST) and then blocked during $2 \mathrm{~h}$ at $37^{\circ} \mathrm{C}$ with PBS containing $2 \%$ of bovine serum albumin (BSA) and washed again 3 times with PBST.

Next, $100 \mu \mathrm{L}$ of biotinylated bovine anti-human IgG-(H+L) (Invitrogen, 1:100, 1:1000 or 1:10000 in PBST-BSA 1\%) or biotinylated goat anti-human IgM-( $\mu$ specific) (Sigma, 1:100, 1:1000 or 1:10000 in PBST-BSA 1\%) secondary antibodies was added and incubated for $1 \mathrm{~h}$ at room temperature (RT). After washing 5 times with PBST, $100 \mu \mathrm{L}$ of high sensitivity streptavidin-HRP (Thermo Scientific, 1:200000 in PBST-BSA 1\%) was added for $30 \mathrm{~min}$ at RT. Subsequently, the plates were washed 5 times with PBST and $100 \mu \mathrm{L}$ of 1-step Ultra TMB (Thermo Scientific) was added for 30 min at RT. Finally, the colorimetric reaction was stopped with $100 \mu \mathrm{L}$ of $2 \mathrm{~N} \mathrm{H}_{2} \mathrm{SO}_{4}$, and absorbance was measured at $450 \mathrm{~nm}$.
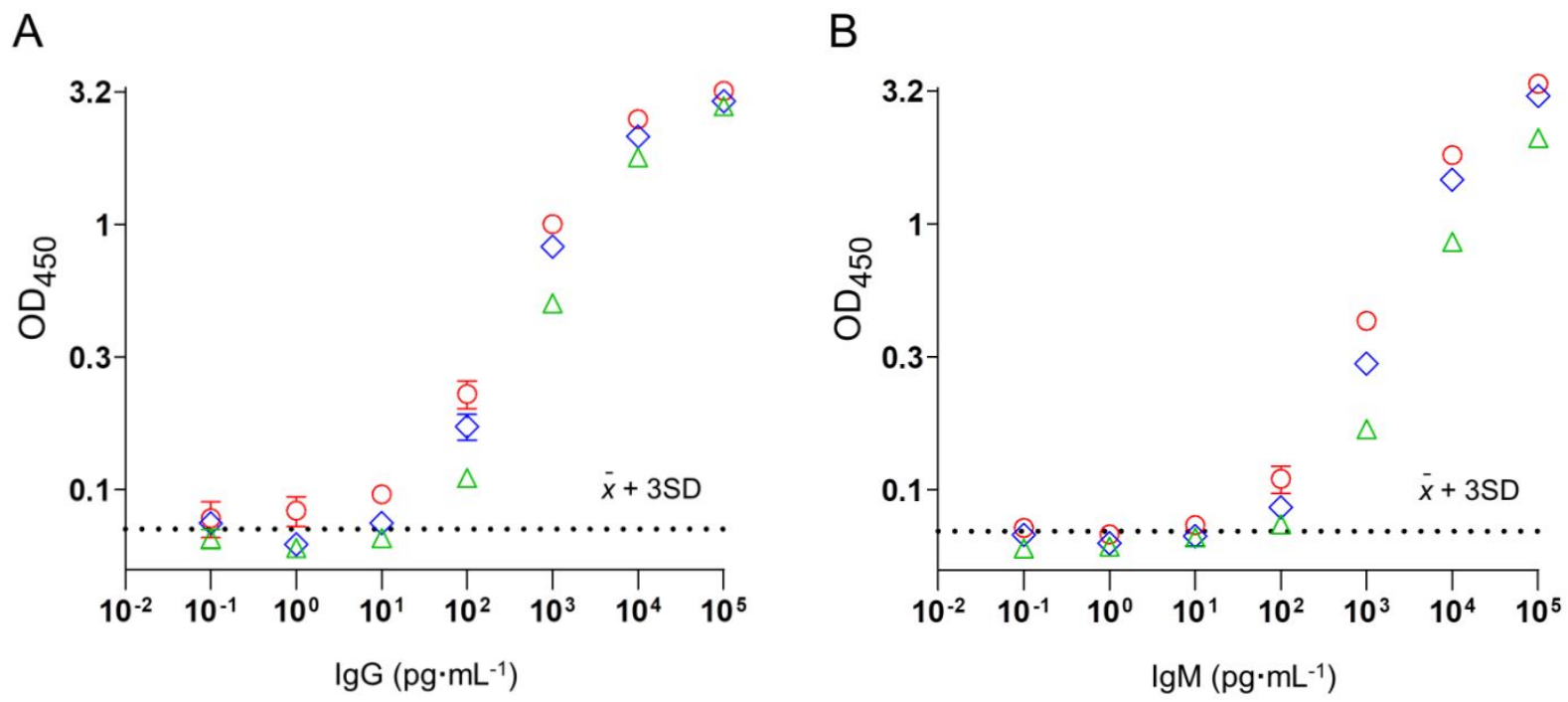

Figure S1. Optimization of secondary biotinylated antibodies to detect human IgM and IgG. Log-log plots representing absorbance with respect to the concentration of $\operatorname{IgG}(\mathrm{A})$ and $\operatorname{IgM}(\mathrm{B})$ using secondary biotinylated antibodies diluted 1:100 (red), 1:1000 (blue) or 1:10000 (green). The dotted line shows the signal above 3 times the standard deviation of the blank. Error bars are the standard deviation $(n=3)$.

Figure S1 shows that the best signal-to-noise ratio was for dilution 1:100 in both cases and the limit of detection, expressed as the assayed sample that yields a signal above three times the standard 
deviation of the blank (99\% confidence), was $1 \mathrm{pg} \cdot \mathrm{mL}^{-1}$ and $10 \mathrm{pg} \cdot \mathrm{mL}^{-1}$ for $\operatorname{IgG}$ and $\operatorname{IgM}$ respectively (Figure $\mathrm{S} 1$ ).

\section{S2. ELISA for comparing the impact of sample pretreatments on immunodetection.}

The following experiment was performed in order to demonstrate that the proposed enzymatic method based on liquefying respiratory samples with catalase-produced oxygen bubbles does not interfere with the recognition function of detection antibodies. Anti-human IgM was treated with DTT or hydrogen peroxide in the presence of catalase. Control experiments were performed with PBS. Then, the antibodies were used to detect human IgM adsorbed onto a microtiter plate. In Figure S2 antibodies incubated with catalase and hydrogen peroxide produce the same colorimetric signal as the PBS control, whereas antibodies treated with DTT generate lower signals. These experiments demonstrate that generation of oxygen bubbles by catalase does not impair the specific recognition function of antibodies.

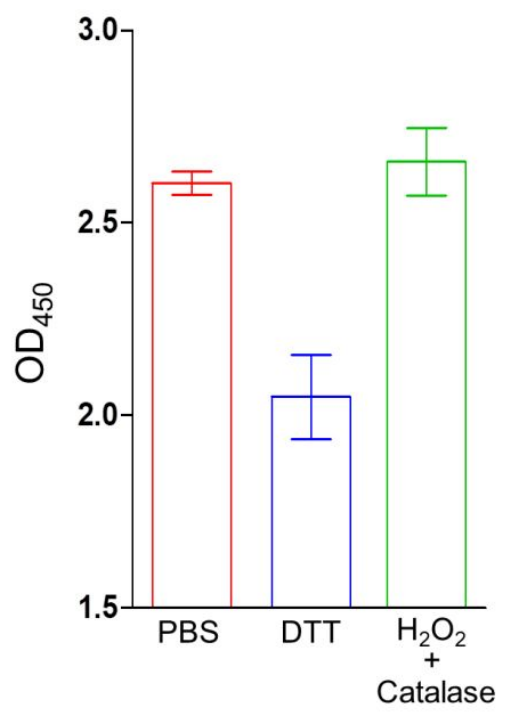

Figure S2. Impact of sample pretreatment by enzymatic method on immunodetection. Colorimetric signals obtained when detecting human IgM-coated wells with secondary biotinylated anti-IgM antibodies previously treated with PBS (red), $6.5 \mathrm{mM}$ DTT (blue) or $0.3 \mathrm{M}$ hydrogen peroxide in the presence of $12.5 \mu \mathrm{M}$ catalase (green). Error bars are the standard deviation $(\mathrm{n}=3)$.

ELISA protocol: the microtiter plate was coated with native human $\operatorname{IgM}\left(10000 \mathrm{pg} \cdot \mathrm{mL}^{-1}, \mathrm{Abcam}\right)$ in bicarbonate buffer $\left(0.1 \mathrm{M}, \mathrm{pH}\right.$ 9.6). After overnight incubation at $4{ }^{\circ} \mathrm{C}$ plates were washed 3 times with PBS containing $0.1 \%$ Tween 20 (PBST), blocked during $2 \mathrm{~h}$ at $37^{\circ} \mathrm{C}$ with PBS containing $2 \%$ of bovine serum albumin (BSA) and then washed again 3 times with PBST. During the blocking step, $10 \mu \mathrm{L}$ of goat anti-human IgM-biotin (Sigma-Aldrich) was treated with $0.3 \mathrm{M}$ hydrogen 
peroxide in the presence of $12.5 \mu \mathrm{M}$ catalase (Sigma-Aldrich), $6.5 \mathrm{mM}$ DTT or PBS for $2 \mathrm{~h}$ at RT (final concentrations in a $30 \mu \mathrm{L}$ reaction). Next, solutions of pretreated goat anti-human IgM-biotin were diluted 1:100 in PBST-BSA 1\% (final antibody dilution 1:300) and $100 \mu \mathrm{L}$ was used as detection antibodies in the previously coated wells with human IgM for $1 \mathrm{~h}$ at RT. Finally, we followed subsequent steps abovementioned in the S1 ELISA protocol 